\title{
Role of Gastrin-17 and Pepsinogen in the Risk Prediction of Gastroesophageal Reflux Disease in the Chinese Population: A Multicenter Clinical Study
}

\author{
Chun-Ping Zhu, Hao-Jie Huang, Jiu-Long Zhao, Jie Chen, Yun-Feng Wang, Yi-Qi Du and Zhao-Shen Li* \\ Department of Gastroenterology, Changhai Hospital, China \\ *Corresponding author: Zhao Shen Li, Department of Gastroenterology, Changhai Hospital, Naval Medical University, 168 \\ Changhai Road, Shanghai 200433, China
}

\begin{abstract}
ARTICLE INFO
Received: 幽 November 19, 2019

Published: 幽 November 27, 2019

Citation: Chun-Ping Zhu, Hao-Jie Huang, Jiu-Long Zhao, Jie Chen, Yun-Feng Wang, Yi-Qi Du, Zhao-Shen Li. Role of Gastrin-17 and Pepsinogen in the Risk Prediction of Gastroesophageal Reflux Disease in the Chinese Population: A Multicenter Clinical Study. Biomed J Sci \& Tech Res 23(2)-2019. BJSTR. MS.ID.003878.
\end{abstract}

Keywords: Gastrin-17; Pepsinogen; Gastro panel; GERD; Diagnosis

Abbreviations: PG: Pepsinogen; PGR: Pepsinogen I/pepsinogen II ratio; GERD: Gastroesophageal reflux disease; BMI: Body mass index; OR: Odds ratio; CI: Confidence interval

\begin{abstract}
Objectives: To determine the usefulness of serum gastrin-17 and pepsinogen examination in the risk prediction of gastroesophageal reflux disease (GERD).

Materials and Methods: This multicenter cross-sectional study involved 6108 patients, of whom 5377 had non-atrophic gastritis (control group) and 731 had GERD. We evaluated the patients' epidemiological data, fasting serum gastrin-17, pepsinogen (PG) I, and PG II levels, and the PG I/PG II ratio (PGR). We performed logistic regression analyses to identify the risk factors for GERD.

Results: Serum gastrin-17 levels decreased in GERD patients, while the PGR increased. When the serum gastrin-17 level was lower than $1.66 \mathrm{pmol} / \mathrm{L}$, the odds ratio (OR) for GERD was 1.35 (95\% CI: 1.15-1.58; P < 0.001). When the PGR was greater than 17 , the OR for GERD was 1.34 (95\% CI: 1.13-1.58; P = 0.001). We identified seven risk factors for GERD: low gastrin-17 level (<1.66 pmol/L), PGR ( $>17)$, male sex, age $\geq 60$ years, body-mass index $\geq 25$, smoking, and clinical reflux symptoms. Helicobacter pylori infection was a protective factor against GERD. The above factors were used to develop a risk-stratification system for GERD, and this system could effectively identify high-risk patients.
\end{abstract}

Conclusion: Serum gastrin-17 level was decreased in patients with GERD, while the PGR was increased. These two parameters combined with other risk factors for GERD could effectively identify patients who were at a high risk for GERD. Serum gastrin-17 and PGR are good indicators for the prediction of GERD risk.

\section{Introduction}

Gastroesophageal reflux disease (GERD) is a common upper gastrointestinal disease. According to the Montreal Consensus [1], GERD is defined as a condition that develops when the gastric contents reflux into the esophagus and cause symptoms and/or complications, which include erosive esophagitis, Barrett esophagus, and non-erosive reflux disease. In recent years, the incidence of GERD has been gradually increasing, and it is 18.1\%-27.8\% in North America and 2.5\%-7.8\% in East Asia [2]. Currently, the diagnosis of GERD mainly involves 24-h pH monitoring, esophageal im pedance monitoring, and endoscopy. However, all these investigations are invasive and painful. Assessment of serum gastrin-17 and pepsinogen levels, also called as GastroPanel in Western countries, is a good indicator of gastric mucosal secretion. They are often used in the diagnosis and screening of gastric diseases such as atrophic gastritis and gastric cancer [3-5]. Gastrin-17 is the amidated form of gastrin, which is synthesized and secreted by the gastric G cells.

The main physiological function of gastrin-17 is to stimulate gastric acid secretion and promote the proliferation and 
differentiation of the gastric mucosal cells. Gastrin-17 accounts for more than $90 \%$ of all the gastrin in the human body [6]. Pepsinogen is an inactive precursor of pepsin. There are two subtypes of pepsinogen, PG I and PG II. PG I is mainly secreted by the chief cells in the gastric fundus and by cervical mucus cells. PG II is secreted from the gastric fundus, gastric pyloric glands, and Brunner glands in the proximal duodenum [7,8]. Pepsinogen levels are an excellent indicator of the morphology and function of the gastric mucosa, and the measurement of these levels is known as a "serological biopsy" [9]. Currently, the relationship of serum gastrin-17 and pepsinogen levels with GERD is not yet clear, especially in the Chinese population. In this study, we aimed to identify the association of serum pepsinogen and gastrin-17 levels with GERD in Chinese patients, in order to determine the usefulness of the assessment of serum gastrin-17 and pepsinogen levels in the risk prediction of GERD.

\section{Materials and Methods}

This multicenter cross-sectional study was from the early gastric cancer screening programmed carried out in the National Center of Clinical Research Center and involved 6108 patients who visited the outpatient departments of 110 top-level hospitals in China between July 2015 and September 2017. Written informed consent to participate in the study was obtained from all participants. Patients were eligible for enrollment if they were 1870 years of age and had been diagnosed with non-atrophic gastritis or GERD (including erosive esophagitis and Barrett esophagus) on gastroscopy and pathological examination. The control group included patients with only chronic non-atrophic gastritis. Patients with other diseases such as peptic ulcer and hiatus hernia were not included, and patients who met any of the following criteria were also excluded from the study:

a) Treatment with drugs such as proton pump inhibitors and $\mathrm{H} 2$ receptor antagonists within 2 weeks before enrollment,

b) History of stomach surgery (including endoscopic surgery), and

c) Severe heart, liver, or renal failure or mental illness.

The following epidemiological data were collected using a questionnaire: age, sex, body mass index (BMI), drinking, smoking, and clinical symptoms of gastric reflux, such as bilious regurgitation and heartburn. Smoking was defined as more than 1 of smoking index (units/day*years). Drinking was defined as more than three meals per week. In this study, endoscopic examination

Table 1: Results of Serum Gastrin-17 and Pepsinogen Assessments.

\begin{tabular}{|c|c|c|c|c|c|c|}
\hline Group & No. of patients & Percentile & Gastrin-17 (pmol/L) & PGR & PG I & PG II \\
\hline \multirow{3}{*}{ Control } & \multirow{3}{*}{5377} & $25 \%$ & 1.2000 & 8.1200 & 74.2950 & 5.2250 \\
\hline & & $50 \%$ & 3.1600 & 12.4000 & 105.6700 & 8.4900 \\
\hline & & $75 \%$ & 9.1875 & 17.1150 & 159.1000 & 15.6000 \\
\hline \multirow{3}{*}{ GERD } & \multirow{3}{*}{731} & $25 \%$ & 1.0000 & 9.1800 & 75.3000 & 5.0800 \\
\hline & & $50 \%$ & $2.6680^{*}$ & $13.0800^{*}$ & 108.3000 & 8.1300 \\
\hline & & $75 \%$ & 8.7000 & 18.6300 & 165.8000 & 14.4700 \\
\hline
\end{tabular}

Copyright@ Zhao-Shen Li | Biomed J Sci \& Tech Res | BJSTR. MS.ID.003878. required at least one chemical staining or electronic staining test based on white light endoscopy. The endoscopic appearance of erosive esophagitis was divided into grades A, B, C, and D according to the Los Angeles classification. For the pathological diagnosis of non-atrophic gastritis, biopsy specimens from the gastric antrum and body were evaluated using a visual analogue scale for chronic gastritis. All patients were instructed to stop taking antacids and gastric mucosa protective agents 2 weeks prior to measurement. After $10 \mathrm{~h}$ of fasting, the patients underwent venous blood collection ( $5 \mathrm{~mL}$ ) in the morning. Enzyme-linked immunosorbent assay (Biohit, Finland) was used to measure the levels of PG I, PG II, gasrin-17, and H. pylori IgG.

\section{Statistics}

Statistical analysis was performed using the SPSS v21.0 software (IBM Corp., Armonk, NY, USA). The serum gastrin-17 and pepsinogen results were expressed as medians, and differences between groups were evaluated using the non-parametric test or chisquare test. The Spearman correlation test was used to determine the relationship of serum gastrin-17 and pepsinogen levels with the risk of GERD. The diagnostic efficacy of serum gastrin-17 and pepsinogen was evaluated using the area under the receiver operating characteristic (ROC) curve. Univariate and multivariate logistic regression analyses were performed to identify the risk factors for GERD. $\mathrm{P}<0.05$ was considered statistically significant.

\section{Ethics}

The patients were enrolled after the study protocol had been approved by the Changhai Hospital Ethics Committee (CHEC2015082).

\section{Results}

A total of 6108 patients who met the selection criteria were enrolled in this study. Endoscopic and pathological examination revealed that of these patients, 5377 had non-atrophic gastritis (control group) and 731 had GERD (GERD group). There were no significant differences between the two groups in terms of age and sex distribution ( $P>0.05$ ). The serum gastrin-17, PG I, and PG II values were not normally distributed, and were therefore expressed as percentiles. The PG I/PG II ratio was higher, and the serum gastrin-17 level was lower in the GERD group than in the control group. The PG I and PG II levels did not significantly differ between the two groups (Table 1). The association of the Los Angeles endoscopic classification of GERD with serum gastrin-17 and the PG I/ PG II ratio was assessed using Spearman correlation coefficients. 
${ }^{*} \mathrm{P}<0.05$, non-parametric test

The Los Angeles endoscopic GERD grade was correlated with both serum gastrin-17 ( $\mathrm{r}=-0.034, \mathrm{P}=0.008)$ and the PG I/PG II ratio $(r=0.041, P<0.001)$. The area under the ROC curve for serum gastrin-17 in the diagnosis of GERD was 0.530 (95\% confidence interval [CI]: 0.506-0.553, P = 0.009), and the optimal cutoff was $1.66 \mathrm{pmol} / \mathrm{L}$. Similarly, the area under the ROC curve for the PG I/PG II ratio in the diagnosis of GERD was 0.538 (95\% CI: 0.5160.560; $\mathrm{P}=0.001$ ), and the optimal cutoff was 17 . In this study, we analyzed the association of the following factors with GERD: serum gastrin-17 levels ( $\leq 1.66 \mathrm{pmol} / \mathrm{L})$, PG I/PG II ratio (>17), sex, age, smoking, drinking, BMI, H. pylori infection, and clinical reflux symptoms (such as regurgitation and heartburn).

According to the results of univariate logistic regression analyses, we identified seven risk factors for GERD: low gastrin-17 level ( $<1.66 \mathrm{pmol} / \mathrm{L})$, PG I/PG II ratio ( $>17)$, male sex, age $\geq 60$ years, BMI $\geq 25$, smoking, and clinical reflux symptoms. H. pylori infection was a protective factor against GERD (Table 2). Factors that were significant on univariate analysis $(\mathrm{P}<0.1)$ were entered into the multivariate analysis: high PG I/PG II ratio $(>17)$, low gastrin-17 (<1.66 pmol/L), male sex, age $>60$ years, BMI $\geq 25$, smoking, drinking, H. pylori infection, and reflux symptoms. Multivariate analysis confirmed that high PG I/PG II ratio (>17), low gastrin-17 (<1.66 pmol/L), male sex, older age, high BMI, smoking, and clinical reflux symptoms were risk factors for GERD (Table 3). We developed a risk-stratification system for GERD based on the $\beta$ value of each variable in the logistic regression model. The total score ranged from 0 to 16 points.

Table 2: Univariate Analysis of Risk Factors for GERD.

\begin{tabular}{|c|c|c|c|}
\hline Risk factor & P value & Odds ratio & $\begin{array}{c}\text { 95\% Confidence } \\
\text { interval }\end{array}$ \\
\hline Male sex & 0.001 & 2.269 & $1.932-2.665$ \\
\hline Age $\geq 60$ years & 0.001 & 1.368 & $1.168-1.603$ \\
\hline BMI $\geq 25$ & 0.001 & 1.456 & $1.222-1.735$ \\
\hline Smoking & 0.001 & 1.922 & $1.617-2.284$ \\
\hline Drinking & 0.006 & 1.323 & $1.085-1.612$ \\
\hline H. pylori infection & 0.002 & 0.776 & $0.660-0.913$ \\
\hline Reflux symptoms & 0.001 & 2.118 & $1.793-2.501$ \\
\hline PGR > 17 & 0.002 & 1.368 & $1.125-1.662$ \\
\hline Low gastrin-17 & 0.001 & 1.351 & $1.153-1.584$ \\
\hline
\end{tabular}

Table 3: Multivariate Analysis of the Risk Factors for GERD.

\begin{tabular}{|c|c|c|c|c|c|c|}
\hline Risk factor & P value & Score & $\boldsymbol{\beta}$ & OR & 95\% CI & \\
\hline \multicolumn{7}{|c|}{ Gender } \\
\hline Female (0) & & 0 & & & & \\
\hline Male (1) & 0.001 & 4 & 0.731 & 2.078 & 1.727 & 2.500 \\
\hline Age (years) & & & & & & \\
\hline$<60$ & & 0 & & & & \\
\hline$\geq 60$ & 0.001 & 2 & 0.333 & 1.395 & 1.186 & 1.640 \\
\hline
\end{tabular}

\begin{tabular}{|c|c|c|c|c|c|c|}
\hline \multicolumn{7}{|c|}{ BMI } \\
\hline$<25$ & & 0 & & & & \\
\hline$\geq 25$ & 0.011 & 1 & 0.223 & 1.249 & 1.043 & 1.496 \\
\hline \multicolumn{7}{|c|}{ Smoking habits } \\
\hline No $(0)$ & & 0 & & & & \\
\hline Yes (1) & 0.024 & 1 & 0.224 & 1.252 & 1.026 & 1.527 \\
\hline \multicolumn{7}{|c|}{ Reflux symptoms } \\
\hline No $(0)$ & & 0 & & & & \\
\hline Yes (1) & 0.001 & 4 & 0.770 & 2.160 & 1.821 & 2.562 \\
\hline \multicolumn{7}{|c|}{ PGR } \\
\hline$<17(0)$ & & 0 & & & & \\
\hline$\geq 17(1)$ & 0.007 & 1 & 0.240 & 1.271 & 1.069 & 1.513 \\
\hline \multicolumn{7}{|c|}{ Gastrin-17 (pmol/L) } \\
\hline$\geq 1.66(0)$ & & 0 & & & & \\
\hline$<1.66(1)$ & 0.002 & 1 & 0.193 & 1.212 & 1.007 & 1.460 \\
\hline$<0.5(2)$ & 0.001 & 3 & 0.503 & 1.653 & 1.286 & 2.125 \\
\hline Constant & -2.855 & & & & & \\
\hline
\end{tabular}

The area under the ROC curve for the risk-stratification system in the diagnosis of GERD was 0.663 (95\% CI: 0.642-0.683; P < 0.001 ), indicating that the system was well differentiated (Table 4). According to the risk scores, the patients were divided into low(0-5 points), moderate- (6-10 points), and high-risk groups (11-16 points). The incidence of GERD in these three groups was $8.0 \%$ (296/3692), 16.0\% (345/2159), and 35.0\% (90/257), respectively. Univariate analysis showed that the ORs for the moderate- and high-risk groups were 2.182 (95\% CI: 1.849-2.575; P < 0.001) and 6.183 (95\% CI: 4.661-8.201; P < 0.001), respectively.

\section{Discussion}

This study showed that in patients with GERD, the serum gastrin-17 level was decreased, while the PG I/PG II ratio was increased. Because GERD is related to gastric acid levels, most patients have high gastric acid levels and a high basal acid output $[10,11]$. Gastric acid inhibits secretion from the G cells, thereby decreasing the serum gastrin-17 level. Gastrin is a gastrointestinal hormone involved in regulating lower esophageal sphincter (LES) function. It promotes LES contraction and propulsive peristalsis in the esophagus by binding to CCK-2 receptors in the LES smooth muscle [12]. Therefore, a decrease in serum gastrin-17 can decrease LES pressure and lead to gastric reflux and GERD [13]. However, we did not assess the relationship between gastrin-17 levels and LES pressure in this study. PG I is a sensitive indicator of the gastric acid level, and increased serum PG I levels and PG I/PG II ratios are associated with increased gastric acid secretion [14,15]. In our study, PG I levels were higher in the GERD group than in the control group, but the difference was not significant.

However, the PG I/PG II ratio was significantly higher in the GERD group. Both gastrin-17 and the PG I/PG II ratio were not very effective in diagnosing GERD, with areas under the ROC curve of 0.530 (95\% CI: 0.506-0.553; P = 0.009) and 0.538 (95\% CI: 0.516- 
0.560; $\mathrm{P}=0.001$ ), respectively. Their optimal cutoffs were 1.66 $\mathrm{pmol} / \mathrm{L}$ and 17, respectively. Sipponen et al. [16] performed a casecontrol study to explore the relationship between serum gastrin-17 and Barrett esophagus. They found that cutoff gastrin-17 values of $0.5 \mathrm{pmol} / \mathrm{L}$ and $1.0 \mathrm{pmol} / \mathrm{L}$ for the diagnosis of Barrett esophagus yielded positive likelihood ratios of 3.5 and 3.0, respectively. The reason for the difference in the gastrin-17 cutoffs between the above study and our study is that only patients with Barrett esophagus were enrolled in the study by Sipponnen et al. Eight risk factors for GERD were identified in this study: high PG I/PG II ratio ( $>17$ ), low gastrin-17 (<1.66 pmol/L), male sex, age $>60$ years, BMI $\geq 25$, drinking, smoking, and reflux symptoms.

In contrast, $H$. pylori infection protected against GERD, which is consistent with previous reports [17]. The reason may be that $H$. pylori-induced gastritis reduces gastric acid secretion [18], thereby reducing the risk of GERD. We also developed a risk-stratification system for GERD based on the above seven factors identified using multivariate logistic regression analysis. This system could effectively identify high-risk patients. Scores of greater than 11 were associated with a significantly increased risk of GERD (OR, 6.183; 95\% CI: 4.661-8.201; $\mathrm{P}<0.001$ ). This study has certain limitations. First, we did not perform 24-h pH monitoring or measure LES pressure, and thus, did not verify the relationship of serum gastrin-17 and pepsinogen with gastric acid levels and LES pressure. Second, the case group in this study included only patients with erosive esophagitis and Barrett esophagus; there were no cases of non-erosive esophagitis.

In conclusion, our results indicate that patients with GERD have a low serum gastrin-17 level and a high PG I/PG II ratio. The riskstratification system could adequately quantify the risk of GERD. Therefore, serum gastrin-17 and PG I/PG II ratio are good indicators for the prediction of GERD risk. We can identify the outpatients who were at a high risk for GERD using the non-invasive method initially.

\section{Disclosure Statement}

No potential conflict of interest was reported by the authors.

\section{Funding}

This study was supported by a grant from the National Clinical Research Center for Digestive Diseases (2015BAI13B08).

\section{Acknowledgment}

a) We thank all the collaborative hospitals in this study.

b) We also thank Medjaden Bioscience Limited for providing assistance in preparing the manuscript.

\section{References}

1. Vakil N, van Zanten SV, Kahrilas P, Dent J, Jones R (2006) The Montreal definition and classification of gastroesophageal reflux disease: a global evidence-based consensus. Am J Gastroenterol 101(8): 1900-1920.
2. El-Serag HB, Sweet S, Winchester CC, Dent J (2014) Update on the epidemiology of gastro-oesophageal reflux disease: a systematic review. Gut 63(6): 871-880

3. Gong Y, Wang W, Li Y, Yuan Yuan (2015) Serum Indicators Reflecting Gastric Function May Also Correlate with Other Extragastric Diseases. Gastroenterol Res Pract: 867495.

4. Korstanje A, den Hartog G, Biemond I, Lamers CB (2002) The serological gastric biopsy: a non-endoscopical diagnostic approach in management of the dyspeptic patient: significance for primary care based on a survey of the literature. Scand J Gastroenterol Suppl 236: 22-26.

5. Adrian G McNicholl, Montserrat Forné, Jesus Barrio, Cristobal De la Coba, Begoña González, et al. (2015) Accuracy of the Serum gastric function test in the detection of atrophic gastritis. Eur J Gastroenterol Hepatol 26(9): 941-948.

6. Mitsutaka Sawada, Chris J Dickinson (1997) The G Cell. Annu Rev Physiol 59: 273-298.

7. Samloff IM (1971) Cellular localization of group I pepsinogens in human gastric mucosa by immunofluorescence. Gastroenterology 61(2): 185188.

8. Samloff IM, Varis K, Ihamaki T (1982) Relationships among serum pepsinogen I, serum pepsinogen II, and gastric mucosal histology: a study in relatives of patients with pernicious anemia. Gastroenterology 83: 204-209.

9. Miki K, Ichinose M, Shimizu A, Huang SC, Oka H, et al. (1987) Serum pepsinogens as a screening test of extensive chronic gastritis. Gastroenterol Jpn 22(2): 133-141.

10. Collen MJ, Johnson DA (1992) Correlation between basal acid output and daily ranitidine dose required for therapy in Barrett's esophagus. Dig Dis Sci 37(4): 570-576.

11. Collen MJ, Johnson DA, Sheridan MJ (1994) Basal acid output and gastric acid hypersecretion in gastroesophageal reflux disease. Correlation with ranitidine therapy. Dig Dis Sci 39(2): 410-417.

12. Liu JF, Gao LP, Wen SW, Lu HL, Zhang J, et al. (2008) Responses of human sling and clasp fibers to cholecystokinin (CCK) and gastrin through CCK receptors. J Gastroenterol Hepatol 23(10): 1608-1612.

13. Vaisrub S (1971) Gastrin and the gastroesophageal sphincter. JAMA 217(8): 1098

14. Samloff IM, Secrist DM, Passaro E Jr (1975) Gastroenterology. 69(6): 1196-1200.

15. Iijima K, Koike T, Abe Y (2014) Cutoff serum pepsinogen values for predicting gastric acid secretion status. Tohoku J Exp Med 232(4): 293300 .

16. Sipponen P, Vauhkonen M, Helske T, Ilpo Kääriäinen, Matti Härkönen (2005) Low circulating levels of gastrin-17 in patients with Barrett's esophagus. World J Gastroenterol 11(38): 5988-5992.

17. Chourasia D, Misra A, Tripathi S, Krishnani N, Ghoshal UC (2011) Patients with Helicobacter pylori infection have less severe gastroesophageal reflux disease: a study using endoscopy, 24-hour gastric and esophageal pH metry. Indian J Gastroenterol 30(1): 12-21.

18. Chourasia D, Ghoshal UC (2008) Pathogenesis of gastro-oesophageal reflux disease: what role do Helicobacter pylori and host genetic factors play? Trop Gastroenterol 29(1): 13-19. 


\section{ISSN: 2574-1241}

DOI: 10.26717/BJSTR.2019.23.003878

Zhao-Shen Li. Biomed J Sci \& Tech Res

(C) This work is licensed under Creative

Submission Link: https://biomedres.us/submit-manuscript.php

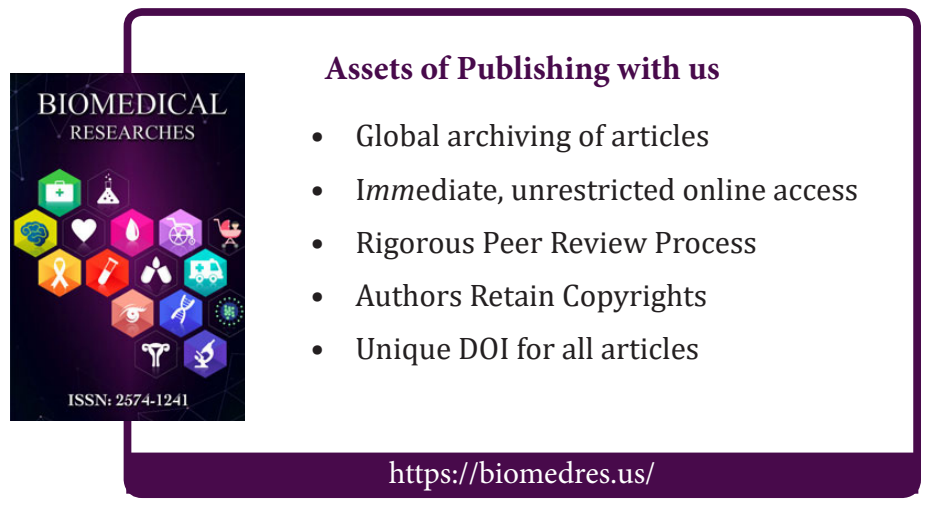

